\title{
BMJ Open Benefits and risks of Iron interventions in children (BRISC): protocol for a three-arm parallel-group randomised controlled field trial in Bangladesh
}

\author{
Mohammed Imrul Hasan, ${ }^{1}$ Sheikh Jamal Hossain, ${ }^{1}$ Sabine Braat, ${ }^{2}$ \\ Michael John Dibley, ${ }^{3}$ Jane Fisher, ${ }^{4}$ Sally Grantham-McGregor, ${ }^{5}$ Fahmida Tofail, ${ }^{6}$ \\ Julie A Simpson, ${ }^{2}$ Shams El Arifeen, ${ }^{1}$ Jena Hamadani, ${ }^{1}$ Beverley-Ann Biggs, ${ }^{7}$ \\ Sant-Rayn Pasricha ${ }^{8}$
}

To cite: Hasan MI, Hossain SJ, Braat S, et al. Benefits and risks of Iron interventions in children (BRISC): protocol for a three-arm parallel-group randomised controlled field trial in Bangladesh. BMJ Open 2017;7:e018325. doi:10.1136/ bmjopen-2017-018325

- Prepublication history for this paper is available online. To view these files, please visit the journal online (http://dx.doi org/10.1136/bmjopen-2017018325).

MIH and SJH contributed equally,

JH, B-AB and S-RP contributed equally.

Received 20 June 2017

Revised 1 September 2017

Accepted 14 September 2017

CrossMark

For numbered affiliations see end of article.

Correspondence to Dr. Sant-Rayn Pasricha; sant-rayn.pasricha@unimelb. edu.au

\section{ABSTRACT}

Introduction Anaemia is a major global health problem affecting about $43 \%$ of preschool children globally and $60 \%$ of 6 -24-month-old children in rural Bangladesh, half of which is attributed to iron deficiency (ID). Although WHO recommends universal supplementation with iron or home fortification with iron-containing multiple micronutrient powders (MMPs) to children under 2 years, evidence for benefits of these interventions on childhood development (a key rationale for these interventions) and harms (especially infection) remains limited. This study aims to evaluate the impact of iron or MMPs supplementation compared with placebo on (a) children's development, (b) growth, (c) morbidity from infections and (d) haematological and iron indices.

Methods and analysis This study is a three-arm, blinded, double-dummy, parallel-group, placebocontrolled superiority trial using stratified individual block randomisation. The trial will randomise 3300 children aged 8-9 months equally to arm 1: iron syrup (12.5 mg elemental iron), placebo MMPs; arm 2: MMPs (including $12.5 \mathrm{mg}$ elemental iron), placebo syrup; and arm 3: placebo syrup, placebo MNPs. Children will receive interventions for 3 months based on WHO recommendations and then be followed up for 9 months post intervention. The primary outcome is cognitive composite score measured by Bayley III. Secondary outcomes include motor and language composite score by Bayley III, behaviour rating using selected items from Wolke's rating scales and BSID-II behaviour ratings, temperament, growth, haemoglobin, anaemia and iron status, and infectious morbidity. Outcomes will be measured at baseline, at the end of 3-month intervention and after 9 months postintervention follow-up.

Ethics and dissemination The trial has been approved by the Ethical Review Committee of icddr,b (Dhaka, Bangladesh) and the Melbourne Health Human Research Ethics Committee (Melbourne, Australia). Results of the study will be disseminated through scientific publications, presentations at international meetings and policy briefs to key stakeholders.

Trial registration number ACTRN12617000660381;Preresults.

WHO Universal Trial Number U1111-1196-1125.

\section{Strengths and limitations of this study}

- Trial design: Double-blind, double-dummy design minimising risk of bias in assessment of outcomes. The trial is designed to be able to compare the main interventions (iron drops and iron-containing micronutrient powders) used for anaemia control in young children against placebo

- Sample size: This is the largest trial to assess effects of iron interventions on child development, and as such, the trial is powered to detect small but clinically relevant effect sizes.

- Trial setting: The trial is set in a low-income South Asian setting where there is a high baseline prevalence of anaemia and will exclude children at risk of high groundwater iron exposure.

- Biomarker assessment: Measurement of anaemia and iron deficiency, along with growth, at baseline will facilitate subgroup analysis by baseline nutrition status.

- We will exclude children with haemoglobin $<8 \mathrm{~g} / \mathrm{dL}$ to ensure they are referred for treatment, which means we will not have a chance to assess the effects of iron interventions on cognitive performance in this group at perhaps higher risk; similarly, children with severe malnutrition are also excluded. Our data may therefore not be able to be generalised to children with severe anaemia or malnutrition.

\section{BACKGROUND}

Anaemia is highly prevalent in preschool children Approximately $43 \%$ (up to 304 million) of under 5 children worldwide are anaemic. The number of children affected is greatest in South Asia, where the prevalence exceeds $55 \%$. $^{1}$ The relative contribution of iron deficiency (ID) to the overall burden of anaemia varies by region. We have previously found that among rural Indian children aged 12-23 months ID accounted for $72 \%$ of anaemia. ${ }^{2}$ In rural Bangladesh, we found about $60 \%$ of 
children 6-24 months to be anaemic, with half of cases due to ID. ${ }^{3}$ Conversely, in preschoolers in rural Gambia and Tanzania where malaria is endemic, ID accounted for only $20 \%$ of anaemia. ${ }^{4}$

\section{Iron supplementation as a strategy for controlling anaemia in children in low-income settings}

Iron supplementation involves administration of medicinal iron (usually ferrous salts). ${ }^{5}$ Multiple micronutrient powders (MMPs) comprise single-dose sachets of lipoencapsulated iron together with other micronutrients (usually at least vitamin A, zinc and folate) that can be sprinkled onto any semisolid food, with the aim of providing a child with a recommended daily intake of micronutrients. WHO recommends two different possible direct interventions for controlling anaemia in young children. First, WHO recommends that all children aged 6-23 months, in settings where the prevalence of anaemia exceeds $40 \%$, receive 3 months daily iron supplements. ${ }^{6}$ Alternatively, where the prevalence of anaemia exceeds $20 \%$, WHO recommends children 6-23 months receive 90 days home fortification with iron-containing MMPs every 6 months. ${ }^{7}$ WHO does not recommend one approach over the other; their efficacy and safety have not been compared in a large head-tohead trial; earlier recommendations for MMPs proposed 2 months intervention every 6 months. Recent estimates indicate that in preschool children about $41 \%$ and $32 \%$ of cases of anaemia in Southeast Asia and sub-Saharan Africa respectively are responsive to iron. ${ }^{8}$

\section{Adequate iron stores are important for neurological development}

The prevalence of anaemia generally increases from 6 months of age and peaks in the second year of life, ${ }^{9}$ especially if iron intake from complementary foods is inadequate to meet the demands of erythropoiesis and growth. ${ }^{11}$ The peak in anaemia prevalence coincides with the critical period for neural development, sharing the same period of peak vulnerability: the 'first 1000 days' from conception to age 2 years. ${ }^{12}$ Animal studies also indicate that iron is needed for myelination and neurotransmitter synthesis, while ID alters neuronal metabolism. ${ }^{13}{ }^{14}$ Observational studies have consistently linked anaemia in infancy to adverse short-term and longer-term deficits in cognitive development. ${ }^{15}$ Hence, animal data and observational studies in children suggest that ID impairs brain development. ${ }^{16}$

\section{Evidence of beneficial effects of iron interventions in children at the population level}

While iron interventions improve haemoglobin $\mathrm{Hb}$ ) concentrations and iron indices and reduce the prevalence of anaemia, ID and iron deficiency anaemia (IDA), ${ }^{17-19}$ there are limited data from population clinical trials confirming that policies of universal iron interventions improve development, growth and health in young children.

\section{Effects on development}

Few randomised controlled trials (RCTs) have evaluated effects of iron supplements or MMPs on development in children under 2 years ${ }^{1820}$ and these trials were underpowered individually and collectively and most of the trials were in preselected patient groups (not populations) or were not blinded (ie, high risk of bias) limiting the quality of evidence. ${ }^{18}$ This paucity of available evidence has hampered systematic reviews and meta-analyses (RCTs) that have to date failed to find evidence of benefit from iron interventions (iron supplements, home fortification with MMPs or other iron interventions) on development in young children. ${ }^{21-24}$ Our systematic review of daily iron supplementation in children aged 4-23 months identified no significant difference in Bayley's mental development index (MDI) in children receiving iron compared with control (mean difference 1.65 (95\% CI -0.63 to 3.94 )); for psychomotor development index the effect size was (mean difference 1.05 (-1.36 to 3.46)). ${ }^{18}$

Systematic reviews evaluating the effects of MMPs on cognitive development did not identify RCTs that had reported effects on measures of cognitive development, ${ }^{25} 20$ and only reported a single trial that found children receiving an intervention walked earlier than those from a parallel control group (ie, children not included in the study at inception). More recently, a large randomised trial in Pakistan identified only transient benefits from MMPs on Bayley's cognitive, language and psychomotor development, ${ }^{26}$ and motor development in the longer term. ${ }^{27}$ This trial did not use placebo and was hence not adequately blinded; moreover, adherence to the supplements appeared limited and had no effect on $\mathrm{Hb}$ concentration compared with control children. ${ }^{28}$

Data regarding longer-term effects of iron supplementation on children development are limited. A recent study in Thailand also documented no significant difference of intelligence quotient and school performance at 9 years of age although the children were supplemented separately with iron and zinc for 6 months at age 4-6 months. ${ }^{28}$ A study in Nepal also found no effect of infant iron supplementation on child's long-term intelligence and executive functions. ${ }^{29}$

\section{Effects on growth}

Benefits on growth are often cited as a rationale for universal iron supplementation. ${ }^{30}$ However, previous systematic reviews have not found benefits on growth, and indeed, have found that iron interventions can impair linear growth in iron-replete children. ${ }^{31}$ Our systematic review suggested daily iron supplementation reduced length and weight gain in young children. ${ }^{18} \mathrm{~A}$ systematic review of iron-containing MMPs found no increase in growth despite containing the growth-promoting micronutrient zinc. ${ }^{20}$

\section{Evidence of harm from iron supplementation}

In contrast to the lack of data on benefits, several large RCTs have reported adverse effects from iron interventions 


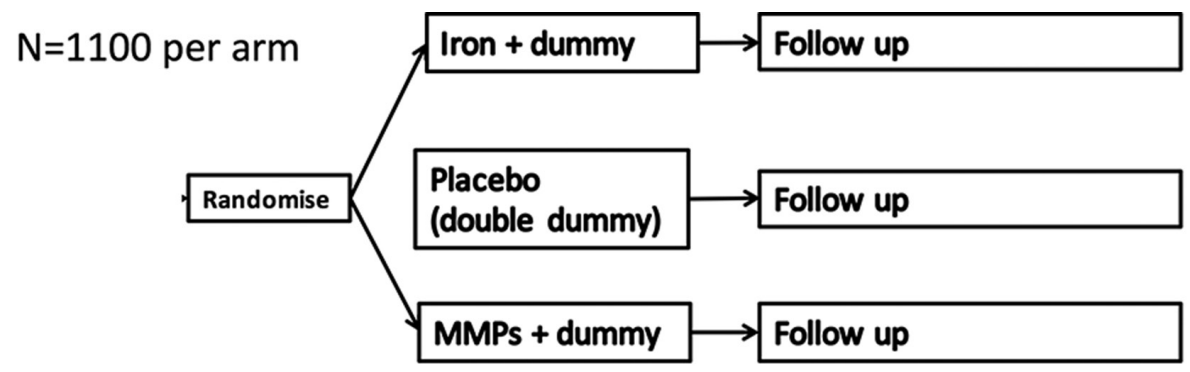

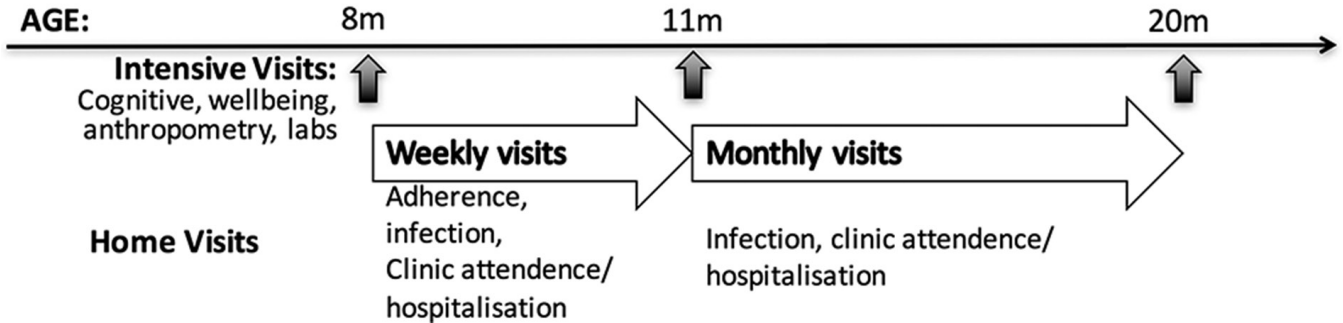

Figure 1 Trial design. MMP, multiple micronutrient powders.

in low-income settings. These emerging data, along with mechanistic studies in low-income settings, are now providing convincing evidence that these interventions cause or exacerbate infection, including diarrhoea, bloody diarrhoea and respiratory infections in endemic and non-endemic malaria settings. ${ }^{26}{ }^{32-34}$ For example, our meta-analysis of iron supplementation identified a $16 \%$ and $38 \%$ increased risk of fever and vomiting, respectively. ${ }^{18}$

\section{The need for a trial}

Although immediate and long-term benefits from iron on functional outcomes such as cognitive development and growth have been assumed for decades, existing data from RCTs do not support this contention. In contrast, data for evidence of harm from iron interventions are accumulating. Furthermore, iron supplements have not been compared directly to MMPs in a large field trial. In this RCT, we aim to define the benefits and harms of daily iron supplementation and MMPs in young children, enabling evidence-based recommendations for implementation (or withdrawal) of iron interventions in this age group.

\section{METHODS AND ANALYSIS}

\section{Benefits and Risks of Iron Interventions in Children}

\section{Trial objectives}

The primary objective of this study is to determine whether 3 months interventions with iron supplementation or home fortification with MMPs is superior to placebo on cognitive development in children aged 8 months \pm 14 days.

The secondary objectives are to evaluate the impact of iron supplementation and home fortification with MMPs, compared with placebo, on
- developmental indices, that is, cognitive (after 9 months post intervention), and motor, language, behaviour and temperament (after 3 months intervention and 9 months post intervention);

- prevalence of anaemia and ID (after 3 months intervention and 9 months post intervention);

- infection risks, especially diarrhoea and respiratory infection in these young children (after 3 months intervention and 9 months post intervention).

\section{Study design}

Benefits and risks of iron interventions in children (BRISC) is a three-arm; parallel; researcher, caregiver, data collector, analysts and participant-blinded-blind; individually randomised; double-dummy placebo-controlled; superiority trial. It will compare the effects of 3 months of daily (a) iron supplementation, or (b) MMPs, to (c) placebo in 8-month-old Bangladeshi children, with a further 9 months follow-up. The trial design is summarised in figure 1.

\section{Study settings and participants}

The trial will be conducted in Rupganj, a rural subdistrict/upazila of Narayanganj district about $50 \mathrm{~km}$ from Dhaka, in Bangladesh. Three unions (regions) within the subdistrict will be included, with each union covered by a dedicated study team. A recent national survey reported the prevalence of anaemia in 9-11-month-old infants at $78.7 \% .^{35}$ Diarrhoea and respiratory infections remain highly endemic in Bangladesh, with $4.6 \%$ and $5.8 \%$ of children $<5$ years experiencing these respectively in a 2-week period.

Like many other developing countries, anaemia is highly prevalent in Bangladesh, and ID is expected to contribute to half the total burden of anaemia ${ }^{312}$ Our study site, Rupganj, is a non-malaria-endemic setting 
in rural Bangladesh and has low groundwater iron level. ${ }^{36}{ }^{37}$ Furthermore, we will exclude any child from a household with elevated groundwater iron. We therefore expect that results from this trial will have generalisability to other low-and-middle-income countries where the prevalence of anaemia is high. This may include malaria-endemic countries; however, the proportion of anaemia attributable to ID in such settings is lower, and the iron-infection interactions may be different. As such, iron trials in malaria-endemic countries should incorporate specific malaria prevention measures which our study does not require. Our study team is also proposing a similar study in Malawi where malaria is endemic, incorporating the requirements for malaria treatment or prevention.

\section{Eligibility criteria}

Children will be randomised only if they fulfil all the inclusion criteria and none of the exclusion criteria.

\section{Inclusion criteria}

1. aged 8 months ( \pm 14 days) at the time of randomisation;

2. not expected to leave the study location for $>1$ week over the next 3 months or for $>1$ month over the next 12 months;

3. has a legal guardian capable of providing informed consent.

\section{Exclusion criteria}

Children meeting any of the following criteria will be excluded from the study:

1. Capillary $\mathrm{Hb}<8.0 \mathrm{~g} / \mathrm{dL}$ at the time of screening.

2. Drinking water iron concentration $>1 \mathrm{mg} / \mathrm{L}$.

3. Diagnosed case of any clinical haemoglobinopathy (eg, beta-thalassaemia major, HbE-beta thalassaemia).

4. Current infective illness (ie, respiratory infection, diarrhoea) with fever; however, children may be rescreened again after recovery if otherwise eligible.

5. Received iron supplements or iron-containing MMP in the previous month.

6. Known congenital anomaly, developmental disorder or severe developmental delay.

7. Child of multiple birth, for example, twins, triplets.

\section{Intervention}

Participants will be randomised in a 1:1:1 ratio to each of the three arms. Infants in the two active intervention arms will receive $12.5 \mathrm{mg}$ daily oral iron either in syrup form or as MMPs as recommended by WHO. ${ }^{38} 39$ Each participant will receive both a syrup (to be dispensed via a syringe at a predefined volume) and a sachet (to be sprinkled on food), achieving double-dummy blinding. Iron syrup and the corresponding placebo will be manufactured in Bangladesh by ACME Laboratories. Micronutrient powders and corresponding placebo will be manufactured by Renata Mothers/caregivers will be instructed (with demonstrations) how to administer the supplements. Participants will be asked to take one dose of each formulation daily for 3 months.
Intervention arms

Arm 1 (iron syrup and placebo sachet): Daily oral supplementation of $12.5 \mathrm{mg}$ elemental iron syrup and a placebo sachet containing powders in identical packaging to the MMP, but containing no micronutrients.

Arm 2 (MMP sachet and placebo syrup): Daily home fortification with an MMP sachet containing $12.5 \mathrm{mg}$ iron, $0.3 \mathrm{mg}$ vitamin $\mathrm{A}, 30 \mathrm{mg}$ vitamin $\mathrm{C}, 0.16 \mathrm{mg}$ folic acid and $5 \mathrm{mg}$ zinc; placebo syrup containing no iron but identical in colour and flavour.

Arm 3 (placebo syrup and placebo sachet): Control arm.

Each participant will receive a pouch every week containing a bottle of syrup and seven sachets.

\section{Randomisation}

Participants will be randomly allocated to one of the three arms with 1:1:1 allocation using a computer-generated schedule of randomly permuted blocks of fixed size stratified by sex and union (each covered by a different field team) to achieve balance between the arms within each stratum. The randomisation list will be prepared by an independent statistician, who will not reveal the block size. The allocation will occur by the field team according to the list, within their assigned union, once eligibility criteria have been checked.

\section{Allocation concealment and blinding of study agents}

Blinding of the team visiting the site, the caregiver(s) and participants will be achieved through the use of identical packaging of sachets and syrup regardless of their contents (active or placebo), packaged in pouches that carry an allocation code. The independent statistician will hold the allocation codes until the data base is ready for unblinding. Researchers, caregivers, persons involved with data collection (ie, field team) or analysis will be blinded to the allocation code until the database has been finalised for analysis. Breaking of the allocation code will occur only in the case of a severe adverse event (AE) or as requested by Data Safety Monitoring Board (DSMB), in which case the code will only be disclosed to the local study physician. Emergency unblinding will lead to discontinuation of the participant's involvement in the study.

\section{Recruitment and visits}

The schedule of visits is outline in table 1. Trained village health workers (VHWs) will identify all potentially eligible children by making household visits in their designated areas and collating these data centrally, enabling generation of a list of age-specific eligible participants in each village. Based on the list, VHWs and senior field assistants (SFAs) will visit potentially eligible families. After providing preliminary information to the parents/guardians, the team will obtain their consent for screening and determine their eligibility. During screening, drinking water iron level will be measured using 'HACH' iron (ferrous) test 
Table 1 Overview of study visits

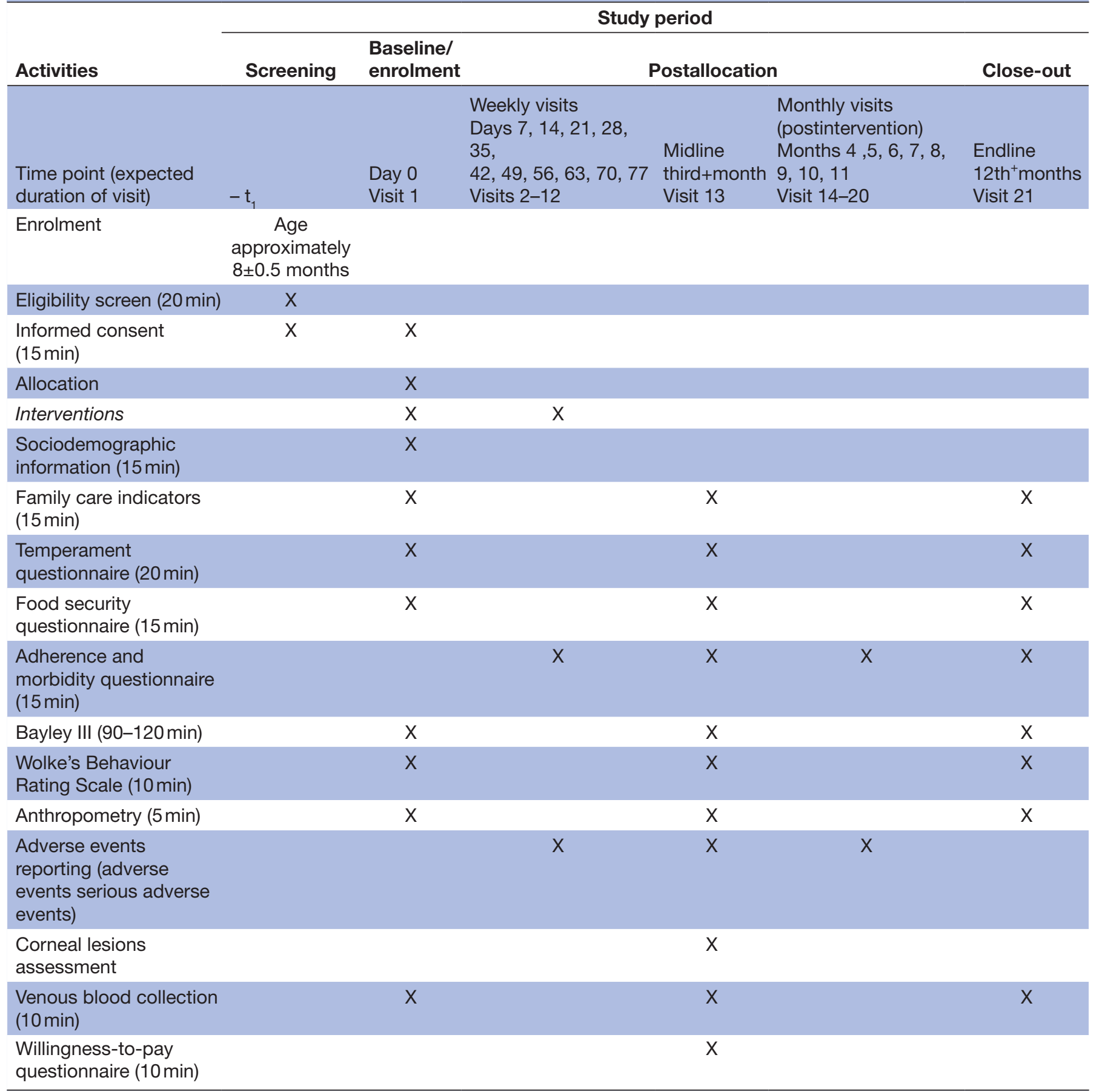

kit and the child's capillary $\mathrm{Hb}$ level will be measured by HemoCue-301. Children with $\mathrm{Hb}<8.0 \mathrm{~g} / \mathrm{dL}$ will be excluded and referred to a nearby health centre for management. Mothers/guardians of eligible children will be briefed further about the trial and be invited to a selected house/test centre for enrolment.

Enrolled families will attend a designated local study site on a prescribed day for enrolment and baseline data collection. The data collection team consisting of a psychological tester, an SFA, a phlebotomist and a VHW will undertake detailed data collection. SFAs will screen children for eligibility, motivate the mothers/ guardians for participation; collect socioeconomic data, household food security and willingness-to-pay (WTP) information. At this visit, consent for participation in the study will be signed by Bayley testers. They will also collect baseline information on child's temperament, family care indicator questionnaire, administer Bayley scale of infant and toddler development (3rd edition), rate the child's behaviour by using Wolke's Behaviour Rating Scale, take anthropometric measurements and, finally, a study phlebotomist will collect $3 \mathrm{~mL}$ of venous blood. The child will then receive the randomly allocated intervention. 
Testers will provide detailed instruction regarding medication to mothers or caregivers before they leave the test centre and they will give details of the enrolled child to the assigned VHW for prospective follow-up visits. The assigned VHW will then visit the child every week for the 3-month intervention period, and every month for the 9-month postintervention period. Morbidity data will be collected weekly and monthly during the intervention and postintervention period, respectively. VHWs will also record and notify any unscheduled hospital or clinic admission experienced by the participant. The number of doses missed by participants will be recorded, empty bottles and sachets will be collected and new doses for the following week dispensed at routine weekly visits. A total of 23 VHWs, 5 SFAs and 10 psychological testers are expected to be recruited and trained for this trial.

\section{Other visits}

Withdrawal visit

Children who stop study drug may continue with assessments if their guardian wishes. If a participant withdraws early or investigator terminates participation, we will seek to undertake the following assessments:

- Reason for study withdrawal.

- If within 2 weeks of visit 13 or 21 , we will invite the participant to attend to undertake this visit unless the reason for withdrawal precludes this.

Recruitment is expected to commence in July 2017 and the trial will be open for 18 months. Expected participant flow is shown in figure 2 .

\section{Study oversight and adherence}

All staff will undergo specific training unique to their role in the study. Adherence will be monitored for all participants. VHWs will measure the amount of syrup and number of sachets unused, and it will be recorded on the case record forms of each child.

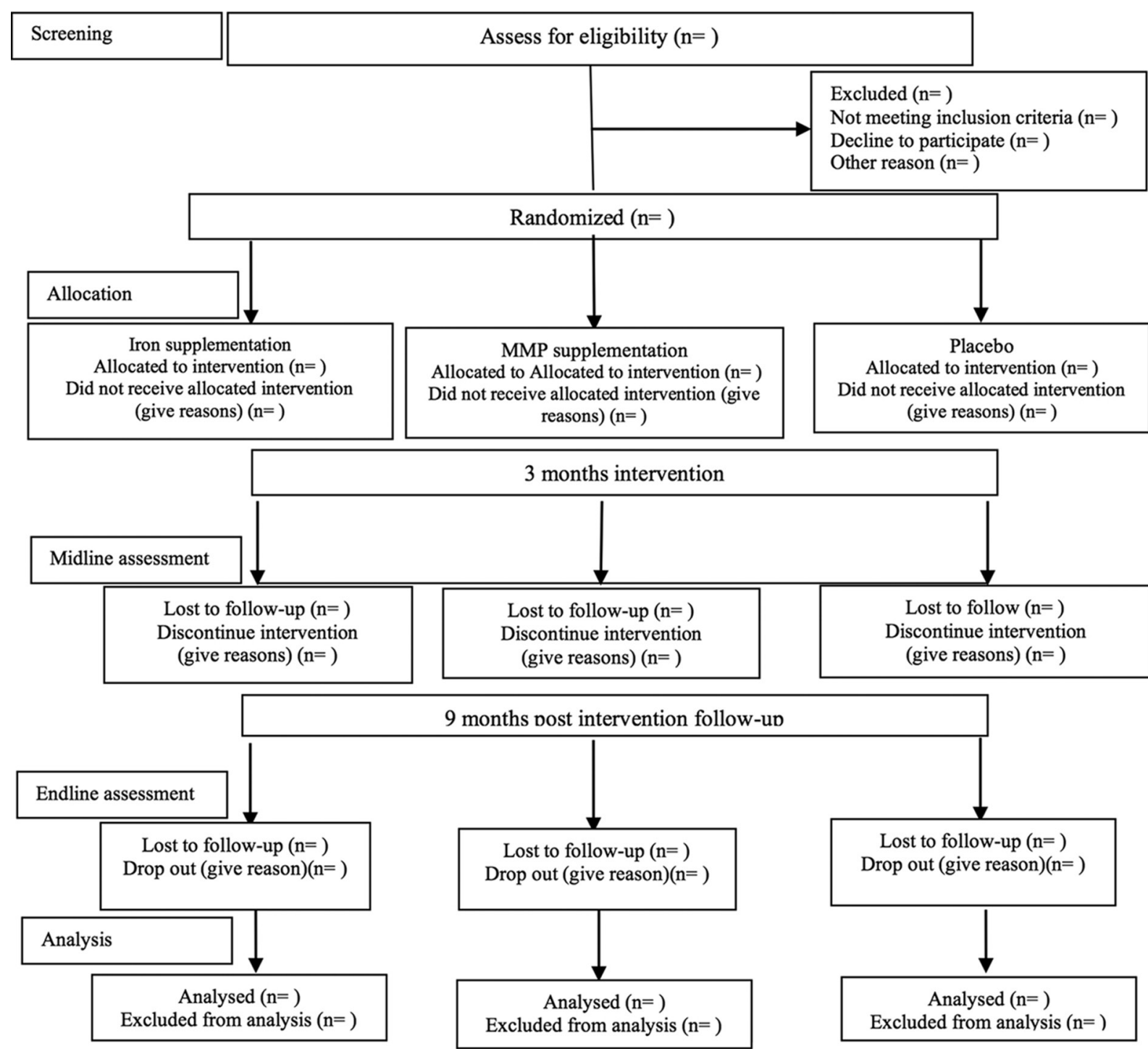

Figure 2 Consolidated Standards of Reporting Trials flow diagram for the Benefits and Risks of Iron Interventions in Children trial. MMPs, multiple micronutrient powders. 


\section{Outcomes}

\section{Primary outcome}

Cognitive composite score (CogCS) measured by Bayley Scales of Infant and Toddler Development (Bayley III) after 3 months of intervention is the primary outcome. ${ }^{40}$ Bayley III is a validated index of child development and the preferred field assessment tool. It is a standard series of measurements primarily to assess cognitive, motor (fine and gross) and language (receptive and expressive) development of infants and toddlers aged $0-31 / 2$ years. Total number of credited items is converted into scaled scores based on child's age, which are then converted to composite scores of each subscale. These scores are used to determine the child's performance compared with norms taken from typically developing children of their age in developed countries. Bayley II has been adapted and extensively used on Bangladeshi children. ${ }^{41-43}$ Bayley -III has now been adapted and some components not familiar for the rural and urban children of this country have been changed according to the local context. It has been used in several studies in this population. ${ }^{44}$ Each tester will receive month-long training for Bayley assessments after employment. Training will cover administration of the testing instruments across all age groups from 1 to 42 months. Refresher training will be provided every 3 months to maintain the consistency and agreements between testers. New testers hired during the course of the study will undergo the same training process. Bayley testers will be certified and permitted to collect data only when their results agree $>90 \%$ with the gold standard, that is, the trainer. About $5 \%-10 \%$ of the tests by each tester will be observed by the trainer for intertester reliability over the course of the study.

\section{Secondary outcomes}

\section{Development}

CogCS at the end of 9 months post intervention, motor and language composite scores by Bayley III, behaviour rating on selected items from Wolke's rating scales and BSID II behaviour ratings, temperament by using a modified version of Bates, quality of home stimulation by using family care indicators and food insecurity by household food insecurity access scale will be measured immediately after intervention and postintervention follow-up. ${ }^{45-48}$ All the secondary outcomes will be assessed at 3 (end of intervention) and 9 months post intervention.

\section{Physical growth}

This will be measured as length, weight, head circumference at end of intervention and post-follow-up period. Length of the child will be measured to the nearest $0.1 \mathrm{~cm}$ by using the Shorr stadiometer (Shorr Products), which has been previously validated and used on local population. Weights will be obtained using a battery-powered digital scale (Tanita HD-318). Length and weight will be used to develop indicators of stunting and wasting compared with age-sex specific WHO international reference growth standards. ${ }^{49}$ Measurements will be taken in duplicate with the average taken unless substantial discrepancy occurs. Physical growth is a secondary outcome of interest, but it can be a confounding factor because malnutrition is correlated to development. We will therefore treat physical growth as both an outcome and a confounder in our analysis. Testers will be certified to take anthropometric measurements only when they achieve a high inter-rater reliability with the trainer, that is, the gold standard. About $5 \%-10 \%$ measures will be checked by the quality assurance team for interobserver reliability throughout the study period. Presence of corneal lesions (caused by vitamin A deficiency) will be assessed by the field team at the midline visit.

\section{Infectious morbidity}

Infectious morbidity as rate and number of days affected by diarrhoea/bloodydiarrhoea (along with number of episodes per day), respiratory infection, vomiting and fever. Infectious morbidity data will be based on previous 7 days recall by caregivers during the 3 months of active intervention. During the subsequent 9 months postintervention follow-up visit, morbidity data will be based on recall for the previous 2 weeks, except for hospitalisation, which will cover previous month's recall. Morbidity information will be collected by VHWs during routine weekly or monthly visits by interviewing caregivers.

\section{Unplanned hospital or healthcare facility attendance}

Unplanned hospital or healthcare facility attendance, as rate, will be measured by field workers along with morbidity questionnaires. Cause-specific attendance will also be ascertained by checking healthcare records by study physician.

\section{Adherence to study medication}

Measured by field workers' audit of packs or measuring the unused doses during weekly visits.

\section{Economic data}

We will collect WTP data to predict the participant's interest and affordability to pay for the price of the intervention. Parent's perception of the benefits (or lack thereof) can also play an important role for uptake of the supplementation. WTP will be measured through the contingent valuation method to predict the maximum price at or below which the participants will definitely buy one unit of the medicine at the end of intervention. Data for future health economic analyses will be collected along with unplanned clinic presentation and hospital admission.

\section{Blood samples}

Three millilitres of venous blood will be collected. Anaemia $(\mathrm{Hb}<11 \mathrm{~g} / \mathrm{dL})$, ID (ferritin $<12 \mathrm{ng} / \mu \mathrm{L}$ ) and IDA (anaemia+ID) will be measured at baseline, at the end of intervention and at postintervention follow-up periods. $\mathrm{Hb}$ will be assessed by HemoCue 301 and ferritin will be assessed by cobas c 311 analyser. Surplus serum and whole blood will be stored for related subsequent studies. 


\section{Sample size and power estimation}

The sample size calculation is based on the primary objective which will be evaluated using the estimated mean difference and $95 \% \mathrm{CI}$ in the change from baseline to 3 months post baseline of the Bayley III CogCS between the iron supplementation and placebo arm, and the MMPs and placebo arm. By construct, the Bayley III CogCS ranges between 55 and 150 (standardised mean 100; SD 15), whereby a higher Bayley III CogCS indicates a better cognitive performance. Our systematic review estimated a difference of 1.65 points $(\mathrm{n}=1093$ across six trials; random-effects 95\% CI (-0.63 to 3.94)) on Bayley MDI (the cognitive scale reported on previous versions of the Bayley scales) in favour of daily iron supplementation compared with control in children aged 4-23 months. Among the six studies included in this systematic review, the highest quality (Cochrane risk of bias tool) study (in Indonesia) evaluating effects on development in a community setting found a two-point difference of universal iron supplementation $(\mathrm{n}=136)$ compared with placebo $(\mathrm{n}=143)$ after 6 months intervention (mean Bayley MDI: iron 101 vs placebo 99, $\mathrm{p}=0.76) .{ }^{50} \mathrm{~A}$ more recent (but non-blinded) trial in Pakistan found a significant 2.5-point difference of MMPs $(\mathrm{n}=658)$ compared with control $(\mathrm{n}=699)$ at 12 months of age (mean Bayley III CogCS: MMPs 95.9 vs placebo 93.4, $\mathrm{p}=0.007) .{ }^{26}$ The sample size required to detect a two-point difference is 883 per arm to reach $80 \%$ power using a two-sided $2.5 \%$ level of significance for each comparison (Bonferroni correction), assuming a 15-point SD. Accounting for about 20\% missing data in Bayley III CogCS at 3 months post baseline, based on a randomised trial in Bangladesh which reported a $26 \%$ loss between birth and 6 months ${ }^{43}$, the total sample size is 3300 . This is currently the largest trial evaluating effects of iron compared with placebo on cognitive development ever to be conducted and will provide evidence for the overall, average effect of these interventions when applied universally to a population with a high prevalence of anaemia, as presently recommended by WHO.

\section{Statistical analysis plan}

All randomised infants will be included in the analysis set according to the arm to which the infant was randomly allocated. Baseline characteristics will be examined across the arms to assess the randomisation. Continuous data will be summarised using mean and SD or median and 25th-75th percentile if data are found to be skewed (eg, ferritin). Categorical data will be presented as count and percentage. The primary outcome, Bayley III CogCS scores at baseline, 3 months and 12 months post baseline, will be analysed using a constrained longitudinal data analysis method. ${ }^{51}$ The model will incorporate time point as a categorical variable and assume a common baseline mean across the three arms. Furthermore, it will adjust for the stratification factors used in the randomisation (gender, union) as main factors and model the variance-covariance among the repeated measurements as unstructured. The estimate and 95\% CI of the mean difference in change from baseline to each postbaseline time point between two arms will be obtained from this model. This model will yield unbiased results when the outcome data are missing at random. In addition, sensitivity analyses consisting of an adjusted analysis accounting for key prognostic baseline variables (eg, socioeconomic status) will be conducted. Secondary continuous outcomes (eg, Bayley III domain scores, anthropometry, z-scores (growth), Behaviour Rating Scale) will be analysed similarly as the primary outcome. Appropriate transformations may be applied to the variables before fitting the model if considered skewed. Secondary binary outcomes (eg, growth stunting, wasting and underweight) will be analysed using generalised estimating equations with a logarithmic link function and unstructured correlation. A Poisson regression, or in case of overdispersion negative binomial regression, will be used to analyse the rate of infections (eg, fever) for the duration of the intervention period, the follow-up period and 12-month study period. The number and percentage of infants with at least one infection, at least one $\mathrm{AE}$ and at least one unplanned hospital or healthcare facility attendance will be tabulated by arm for the duration of the intervention period, the follow-up period and 12-month study period. A per-protocol analysis of efficacy outcomes, based on adherence, and as as-treated analysis of safety outcomes, in case of misrandomisation, will also be conducted. Exploratory subgroup analyses will be performed irrespective of the primary study findings by (a) baseline anaemia status (yes vs no anaemia), (b) baseline ID status (yes vs no iron deficient), (c) baseline ID anaemia status (yes vs no iron-deficient anaemia), (d) baseline home stimulation (above vs below median level as measured by family care indicators), (e) wealth status (above or below median), (f) growth (presence or absence of stunting), (g) infant's sex (male vs female) and (h) food security status, by adding subgroup as a main effect and its interaction with treatment arm to the model to evaluate whether the treatment effect differs across subgroup categories. We postulate that infants with anaemia, ID, IDA or above median home stimulation will have a larger treatment effect compared with those whom are non-anaemic, non-iron deficient, non-iron-deficient anaemic or below median home stimulation, respectively.

In addition, depending on the findings of the study, we will undertake subsequent health economics analysis of the data. For this purpose, we will collect and present all direct and indirect costs for the implementation of the project. The contingent valuation methods will be used to estimate weekly WTP and multiple regression analysis will be used to predict WTP by socioeconomic characters, past illness and type of medicine.

\section{Data management}

Data from questionnaires will be entered directly into electronic tablets in the field, along with Global Positioning System location data. Data will be checked in real time 
for quality by a dedicated data manager. Data for Bayley scales will be entered subsequently, with $10 \%$ undergoing double entry. Range checks will be applied automatically to all data. All aspects of the trial conduct (field work, eg, ethical recruitment and consent, randomisation, provision of interventions, outcome assessments, data collection and entry) will be audited at least annually by investigators from the University of Melbourne.

\section{ETHICS AND DISSEMINATION}

A placebo-controlled trial is essential to establish the efficacy and adverse effects of iron on children's health and development, and is considered ethically justifiable because (a) there is uncertainty regarding the benefits of iron supplementation on cognitive function; (b) all families will be educated about iron nutrition; (c) children with anaemia at the final measurement $(+12 \mathrm{~m})$ will be referred to health centres; (d) there is previous experience of use of placebo arm in large iron/MMP RCTs, for example, in Tanzania, Ghana, Nepal and many other countries; and (e) mild-moderate ID is not yet known to cause, and iron interventions to alleviate, moderate or severe, permanent cognitive delay. Even though universal supplementation is recommended by WHO, it is not yet practised in Bangladesh.

The trial has been approved by the Melbourne Health Human Research Ethics Committee, Australia (2016.269); the Ethical Review Committee of icddr,b (PR-16063); and the Directorate General of Drug Administration, Ministry of Health and Family Welfare, Bangladesh. Informed written consent will be obtained from parents/guardians prior to both screening and enrolment procedureseither via signature or a thumbprint or mark for those who cannot signs. Written informed consent from the child's parent or legal guardian will be obtained by the SFA, the most senior member of the field data collection teams. Consent will encompass participation in the trial and its procedures, as well as storage and possible use of samples for related studies in the future; this includes non-diagnostic molecular and genetic studies. Children ineligible at recruitment due to illness will be referred for clinical care. Any information obtained in connection with this research project or in any publication and/ or presentation will be provided in such a way that the individual cannot be identified. Only researchers on this project will have access to the data. Three years after the protocol completion date, icddr,b research data in the repository will be made publicly available according to icddr,b data access policy.

\section{Data monitoring}

An independent DSMB has been constituted and will provide oversight of the study. In cases of serious AEs, the study physician will follow-up and document the course of events, will recommend for necessary suspension, refer if necessary and report to DSMB. As per best practice, the DSMB will define their meeting schedule and plan for interim analyses and define stopping rules in the DSMB charter. Amendments to the trial protocol will be updated in the trial protocol, the trial registration, informed by memo to all investigators, as well as the ethical review committees, and if significant, will be explained in the final publications of the trial.

\section{Discussion}

Understanding the benefits and risks of universal iron interventions in young children at the population level is a public health priority. This pivotal trial will form the platform for global anaemia control policy in young children. It will define global guidelines, inform policymakers at the national and regional level, and provide the economic rationale for donors and governments to select and fund anaemia control interventions. The design (combining interventions with vaccination) will enable translation to the field. Results will be communicated to the academic community through publication in peer-reviewed journals. Criteria for authorship will reflect International Committee of Medical Journal Editors guidelines. We will also communicate results to policymakers through policy briefs and reports, for example, WHO, Unicef and major nutrition bodies (eg, GAIN).

\section{Author affiliations}

${ }^{1}$ Maternal and Child Health Division, International Centre for Diarrhoeal Disease Research, Dhaka, Bangladesh

${ }^{2}$ Centre for Epidemiology and Biostatistics, Melbourne School of Population and Global Health and Melbourne Clinical and Translational Sciences (MCATS), University of Melbourne, Melbourne, Australia

${ }^{3}$ School of Public Health, University of Sydney, Sydney, Australia

${ }^{4}$ School of Public Health and Preventive Medicine, Monash University, Melbourne, Australia

${ }^{5}$ Centre for International Child Health, University College London, London, UK ${ }^{6}$ Nutrition and Clinical Services Division, International Centre for Diarrhoeal Disease Research, Dhaka, Bangladesh

${ }^{7}$ Department of Medicine, Doherty Institute, Melbourne, Australia

${ }^{8}$ Department of Medicine, The Royal Melbourne Hospital, Melbourne, Australia

Contributors SP and BB conceived of the idea for the trial. SP, BB, MD, JF, SGM, JS, SA and JH prepared the initial funding submissions and proposals. MIH, SJH, $\mathrm{SB}, \mathrm{JH}, \mathrm{SP}$ and BB prepared the detailed trial protocol. SB and JAS developed the statistical analysis plan. MOH, SJH, FT and JH designed the field work. MIH, SJH and SP wrote the first draft of the manuscript, and all authors have reviewed and authorised it.

Funding The BRISC trial is being implemented by icddr,b in collaboration with University of Melbourne and funded by NHMRC, grant number 1103262. The trial is investigator initiated and sponsored, the trial sponsor will be the University of Melbourne.

Competing interests None declared.

Provenance and peer review Not commissioned; externally peer reviewed.

Open Access This is an Open Access article distributed in accordance with the Creative Commons Attribution Non Commercial (CC BY-NC 4.0) license, which permits others to distribute, remix, adapt, build upon this work non-commercially, and license their derivative works on different terms, provided the original work is properly cited and the use is non-commercial. See: http://creativecommons.org/ licenses/by-nc/4.0/

(C) Article author(s) (or their employer(s) unless otherwise stated in the text of the article) 2017. All rights reserved. No commercial use is permitted unless otherwise expressly granted. 


\section{REFERENCES}

1. Stevens GA, Finucane MM, De-Regil LM, et al. Global, regional, and national trends in haemoglobin concentration and prevalence of total and severe anaemia in children and pregnant and nonpregnant women for 1995-2011: a systematic analysis of populationrepresentative data. Lancet Glob Health 2013;1:e16-25.

2. Pasricha SR, Black J, Muthayya S, et al. Determinants of anemia among young children in rural India. Pediatrics 2010;126:e140-9.

3 Tofail F. Prevalence of iron deficiency anaemia among young children in rural Bangladesh. Health and Science Bulletin 2010;8:1-6.

4. Pasricha SR, Atkinson $\mathrm{SH}$, Armitage AE, et al. Expression of the iron hormone hepcidin distinguishes different types of anemia in African children. Sci Transl Med 2014;6:235re3.

5. Pasricha SR, Flecknoe-Brown SC, Allen KJ, et al. Diagnosis and management of iron deficiency anaemia: a clinical update. Med $J$ Aust 2010;193:525-32.

6. WHO. Daily iron supplementation in children 6-23 months of age. e-Library of Evidence for Nutrition Actions (eLENA), 2016. http:// www.who.int/elena/titles/guidance_summaries/iron_children/en/ (accessed 11 Jul 2016).

7. WHO. WHO guideline: Use of multiple micronutrient powders for point-of-use fortication of foods consumed by infants and young children aged 6-23 months and children aged 2-12 years. Geneva: World Health Organization, 2016.

8. World Health Organization. The global prevalence of anaemia in 2011. Geneva: World Health Organization, 2015.

9. Black RE, Allen LH, Bhutta ZA, et al. Maternal and child undernutrition: global and regional exposures and health consequences. Lancet 2008;371:243-60.

10. Pasricha SR. Anemia: a comprehensive global estimate. Blood 2014;123:611-2.

11. Pasricha SR, Drakesmith $\mathrm{H}$, Black J, et al. Control of iron deficiency anemia in low- and middle-income countries. Blood 2013;121:2607-17.

12. Kassebaum NJ, Jasrasaria R, Naghavi M, et al. A systematic analysis of global anemia burden from 1990 to 2010. Blood 2014;123.

13. Beard J. Recent evidence from human and animal studies regarding iron status and infant development. J Nutr 2007;137:524S-30.

14. Beard JL. Why iron deficiency is important in infant development. $J$ Nutr 2008;138:2534-6.

15. McCann JC, Ames BN. An overview of evidence for a causal relation between iron deficiency during development and deficits in cognitive or behavioral function. Am J Clin Nutr 2007;85:931-45.

16. Walker SP, Wachs TD, Gardner JM, et al. Child development: risk factors for adverse outcomes in developing countries. Lancet 2007;369:145-57.

17. Low M, Farrell A, Biggs BA, et al. Effects of daily iron supplementation in primary-school-aged children: systematic review and meta-analysis of randomized controlled trials. CMAJ 2013;185:E791-802.

18. Pasricha SR, Hayes E, Kalumba K, et al. Effect of daily iron supplementation on health in children aged 4-23 months: a systematic review and meta-analysis of randomised controlled trials. Lancet Glob Health 2013;1:e77-86.

19. Thompson J, Biggs BA, Pasricha SR. Effects of daily iron supplementation in 2- to 5-year-old children: systematic review and meta-analysis. Pediatrics 2013;131:739-53.

20. De-Regil LM, Suchdev PS, Vist GE, et al. Home fortification of foods with multiple micronutrient powders for health and nutrition in children under two years of age. Cochrane Database Syst Rev 2011;9:CD008959.

21. Sachdev H, Gera T, Nestel P. Effect of iron supplementation on mental and motor development in children: systematic review of randomised controlled trials. Public Health Nutr 2005;8:117-32.

22. Szajewska H, Ruszczynski M, Chmielewska A. Effects of iron supplementation in nonanemic pregnant women, infants, and young children on the mental performance and psychomotor development of children: a systematic review of randomized controlled trials. Am J Clin Nutr 2010;91:1684-90.

23. Wang B, Zhan S, Gong T, et al. Iron therapy for improving psychomotor development and cognitive function in children under the age of three with iron deficiency anaemia. Cochrane Database Syst Rev 2013;6:CD001444.

24. Horton S, Ross J. The economics of iron deficiency. Food Policy 2003;28:51-75.

25. Larson LM, Yousafzai AK. A meta-analysis of nutrition interventions on mental development of children under-two in low- and middleincome countries. Matern Child Nutr 2017;13:e12229.

26. Yousafzai AK, Rasheed MA, Rizvi A, et al. Effect of integrated responsive stimulation and nutrition interventions in the lady health worker programme in Pakistan on child development, growth, and health outcomes: a cluster-randomised factorial effectiveness trial. Lancet 2014;384:1282-93.

27. Yousafzai AK, Obradović J, Rasheed MA, et al. Effects of responsive stimulation and nutrition interventions on children's development and growth at age 4 years in a disadvantaged population in Pakistan: a longitudinal follow-up of a cluster-randomised factorial effectiveness trial. Lancet Glob Health 2016;4:e548-58.

28. Pongcharoen T, DiGirolamo AM, Ramakrishnan U, et al. Long-term effects of iron and zinc supplementation during infancy on cognitive function at 9 y of age in northeast Thai children: a follow-up study. Am J Clin Nutr 2011;93:636-43.

29. Christian P, Morgan ME, Murray-Kolb L, et al. Preschool iron-folic acid and zinc supplementation in children exposed to iron-folic acid in utero confers no added cognitive benefit in early school-age. $J$ Nutr 2011;141:2042-8.

30. WHO/UNICEF/UNU. Iron Deficiency Anaemia: Assessment, Prevention, and Control. A guide for programme managers. Geneva: World Health Organization, 2001.

31. Sachdev H, Gera T, Nestel P. Effect of iron supplementation on physical growth in children: systematic review of randomised controlled trials. Public Health Nutr 2006;9:904-20.

32. Sazawal S, Black RE, Ramsan M, et al. Effects of routine prophylactic supplementation with iron and folic acid on admission to hospital and mortality in preschool children in a high malaria transmission setting: community-based, randomised, placebo-controlled trial. Lancet 2006;367:133-43.

33. Soofi S, Cousens S, Iqbal SP, et al. Effect of provision of daily zinc and iron with several micronutrients on growth and morbidity among young children in Pakistan: a cluster-randomised trial. Lancet 2013;382:29-40.

34. Zlotkin S, Newton S, Aimone AM, et al. Effect of iron fortification on malaria incidence in infants and young children in Ghana: a randomized trial. JAMA 2013;310:938-47.

35. National Institute of Population Research and Training (NIPORT) MaA all. Bangladesh - Demographic and Health Survey 2011. Dhaka, Bangladesh and Calverton, Maryland, USA: NIPORT, Mitra and Associates, and ICF International, 2013.

36. Haque U, Overgaard HJ, Clements AC, et al. Malaria burden and control in Bangladesh and prospects for elimination: an epidemiological and economic assessment. Lancet Glob Health 2014;2:e98-105.

37. Kinniburgh D, Smedley P. Arsenic contamination of groundwater in Bangladesh, 2001.

38. WHO Guidelines Approved by the Guidelines Review Committee. Guideline: Use of Multiple Micronutrient Powders for Home Fortification of Foods Consumed by Infants and Children 6-23 Months of Age. Geneva: World Health Organization, 2011.

39. WHO Guidelines Approved by the Guidelines Review Committee. Guideline: Daily Iron Supplementation in Infants and Children. Geneva: World Health Organization, 2016.

40. Bayley N. Bayley Scales of Infant and Toddler Development: Motor Scale. Psychological Corporation, 2006.

41. Hamadani JD, Baker-Henningham $\mathrm{H}$, Tofail F, et al. Validity and reliability of mothers' reports of language development in 1-yearold children in a large-scale survey in Bangladesh. Food Nutr Bul 2010;31:S198-206.

42. Tofail F, Persson LA, El Arifeen S, et al. Effects of prenatal food and micronutrient supplementation on infant development: a randomized trial from the Maternal and Infant Nutrition Interventions, Matlab (MINIMat) study. Am J Clin Nutr 2008;87:704-11.

43. Parveen M, Rahman ST, Islam S, et al. Adaptation of items of bayley scales of infant development-II (BSID-II) suitable for Bangladeshi infants. Dhaka University Journal of Biological Sciences 2014;23:187-95.

44. Jiang NM, Tofail F, Moonah SN, et al. Febrile illness and pro-inflammatory cytokines are associated with lower neurodevelopmental scores in Bangladeshi infants living in poverty. BMC Pediatr 2014;14:50.

45. Wolke D, Skuse D, Mathisen B. Behavioral style in failure-tothrive infants: a preliminary communication. J Pediatr Psycho 1990;15:237-54.

46. Bayley N. Manual for the Bayley Scales of Infant Development II. San Antonio, TX: The Psychological Corporation, 1993.

47. Coates J, Swindale A, Bilinsky P. Household Food Insecurity Access Scale (HFIAS) for Measurement of Food Access: Indicator Guide v. 3. Washington, DC: Food and NutritionTechnical Assistance Project (FANTA), Academy for Educational Development, Google Scholar, 2007

48. Hamadani JD, Tofail F, Hilaly A, et al. Use of family care indicators and their relationship with child development in Bangladesh. $J$ Health Popul Nutr 2010;28:23-33. 
49. World Health Organization. WHO child growth standards: length/ height for age, weight-for-age, weight-for-length, weight-for-height and body mass index-for-age, methods and development: World Health Organization, 2006.

50. Lind T, Lönnerdal B, Stenlund $\mathrm{H}$, et al. A community-based randomized controlled trial of iron and zinc supplementation in
Indonesian infants: effects on growth and development. Am J Clin Nutr 2004;80:729-36.

51. Ward DG, Roberts K, Stonelake P, et al. SELDI-TOF-MS determination of hepcidin in clinical samples using stable isotope labelled hepcidin as an internal standard. Proteome Sci 2008;6:28. 